\title{
Applications of Embedded Controllers in Speed and Position Controls
}

\author{
Chiu H. CHOI, Ph.D., P.E. \\ Electrical Engineering Program \\ University of North Florida \\ Jacksonville, FL 32224, USA \\ Email: cchoi@unf.edu
}

\begin{abstract}
This tutorial paper presents the experimental results of solving speed and position control problems by using embedded controllers. The embedded controllers used proportional, integral, and derivative controls. A feature of these controllers is that the sample time is not explicitly included in the integral and derivative control equations. This feature was experimentally tested. In the testing of the integral control with such feature, the controller was applied to a speed regulation problem under the condition of rapidly changing load. In the testing of the derivative control with the same feature, the controller was applied to a ringing removal problem. The results were that these controllers performed as expected. Other experiments of position controls and their results were also presented. All the embedded controllers were implemented in a member of Freescale Semiconductor's MC68HC12 microcontroller family.
\end{abstract}

\section{INTRODUCTION}

Speed and position control problems are among the common control problems arising in industrial controls and many disciplines of engineering. Many theoretical and experimental treatments for these problems have been proposed and can be found in the literature [119]. A widely used approach to solving the speed and position control problems is to use compensators such as proportional controllers, proportional-derivative controllers, proportional-integral controllers, and PID controllers. However, it is not a simple task to select the correct gains theoretically. The best gains are sometimes found in an experimental manner. This is the approach taken in this paper.

These compensators can be implemented in various forms such as operational amplifier circuits or as $\mathrm{C}$ or assembly language programs running on an embedded processor. In this paper, the compensators are implemented as $\mathrm{C}$ programs running on an embedded processor, in particular the Freescale MC68HC912B32 microcontroller. Such compensators can be coded as a stand alone $\mathrm{C}$ program and be put to work quickly. A feature of these controllers considered in this paper is that the sample time is not explicitly included in the derivative and integral control terms.

The equipment used in testing the workings of the embedded controllers covered in this paper consist of an Axiom CME12B evaluation board for MC68HC912B32, an Image Craft ICC12 C compiler, signal conditioning circuits consisting of operational amplifiers and resistors, a Feedback mechanical unit model 33-100, and a Tiepie virtual oscilloscope model HS-3 running on a Pentium PC.

The Axiom board and the $\mathrm{C}$ compiler are used for implementing the compensators into the microcontrollers as embedded controllers.

Signal conditioning circuits were used to convert the electrical signals from the Feedback mechanical unit into a voltage range within the specification of the onchip analog-to-digital (ATD) converters in the microcontroller. The conversion was from the voltage range of $-10 \mathrm{~V}$ to $+10 \mathrm{~V}$ into the range of $0 \mathrm{~V}$ to $+5 \mathrm{~V}$ in a linear fashion. The latter range was the range that was acceptable by the ATD converters. The resolution of the ATD converters was chosen to be eight bit.

The Feedback mechanical unit consists of an input commanding potentiometer connected to a dial, an output following potentiometer connected to a shaft driven by a permanent magnet DC motor and associated belt drive system. The motor is driven by a power amplifier housed inside the unit. A tachometer connected to the output shaft is available. There is a magnetic brake in the unit. There are also other features in the unit but they are not used in the experiments covered in this paper.

The Tiepie virtual oscilloscope was used to record the input and output signals generated in the testing of the controllers.

The configuration of the embedded control system under consideration is shown in Figure 1. The ATDs, the summer, and the controller with its pulse-width modulated output signal belong to the microcontroller. 
The summer and the controller were implemented as software running inside the microcontroller. The pulsewidth modulated signal was generated by an on-chip pulse-width modulator.

The components in the rest of Figure 1 except the signal conditioning circuits belong to the Feedback mechanical unit. The pulse-width modulated signal drives the power amp (which is an H-bridge) and the motor gear in the following particular manner.

- If the duty cycle of the pulse-width modulated signal is greater than $50 \%$, the motor turns counter-clockwise.

- If the duty cycle is less than $50 \%$, the motor turns clockwise.

There are other educational products that house all these components together as one unit. For instance, Quanser's QET is one such product. It was the Feedback's unit that was used as the plant in each of the embedded control problems considered in this paper.

The embedded control problems under consideration are briefly described as follows: the first problem is a speed regulation problem under the condition of rapidly varying load. The second problem is a position tracking problem that an embedded controller is designed to drive the output shaft to track the position of the input dial. The third problem is to design a controller that drives the output shaft to mirror the position of the input dial. The fourth problem is to design a controller that drives the output shaft to lead the input dial by a fixed angle. The last problem is to design a controller that eliminates the ringing of the second problem when excessive gain is used to drive the output shaft.

The rest of this paper is organized as follows: in the next section the embedded control solution to each problem are described and the experimental results are presented. Section III provides some concluding remarks.

\section{EMBEDDED CONTROLLER SOLUTIONS}

In this section five control problems are stated and their solutions using embedded controllers are described. Experimental results showing the workings of these embedded controllers are also provided.

Problem 1: Use the $\mathrm{HC} 12$ microcontroller as an embedded controller to drive a DC motor at a constant speed regardless of the amount of loading on the motor.
A block diagram for a solution to this design problem is shown in Figure 2. Vref is the set point. It corresponds to a constant created in the $\mathrm{C}$ program for the speed controller. Vtacho is the digitized tachometer voltage. It is proportional to the motor speed. Ve is the error, which drives the controller. The summer and the controller are implemented as a $\mathrm{C}$ program running inside the embedded controller.

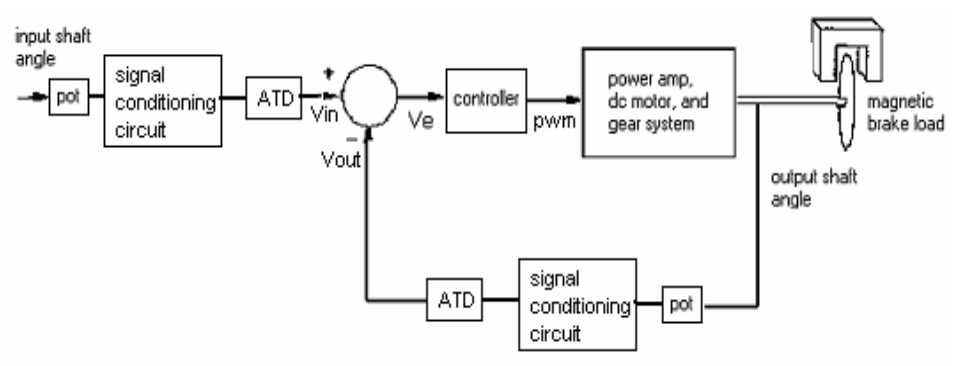

Figure 1: Block diagram of the embedded control system

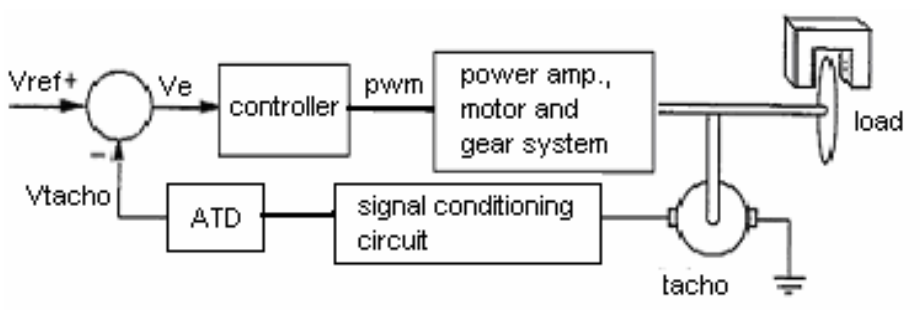

Figure 2: Block diagram of Problem 1

The controller can be a PI controller, a PID controller or simply an integrator. These controllers eliminate the steady state error. The PI or PID controller will normally produce less ringing in the response than the integrator but requires more computations. The controller chosen to solve this problem was a PI controller. The duty cycle of the pulse-width modulated signal generated in the on-chip pulse-width modulator is governed by the expression

$\mathrm{Kp} * \mathrm{Ve}+\mathrm{Ki}^{*} \int_{0}^{t} V_{e}(u) d u$, where the constant $\mathrm{Kp}$ is the proportional gain factor. The constant $\mathrm{Ki}$ is the integral gain factor.

The PI controller equation implemented in the $\mathrm{C}$ program are shown below. These equations are executed once every $\mathrm{T}$ seconds, where $\mathrm{T}$ is the sample time. The signals Ve, Vref, and Vtacho are shown in Figure 2.

$\mathrm{Ve}=($ Vref - Vtacho $) ;$ integral = integral + Ve; $\mathrm{n}=(((-\mathrm{Ve} * \mathrm{Kp}-\mathrm{Ki} *$ integral $) * 0.5 / 255)+0.5) * 100$; 
Notice that the sample time $\mathrm{T}$ is not explicitly shown in the second equation for the integral. Strictly speaking, $\mathrm{T}$ should be included. However, the sample time $\mathrm{T}$ can always be combined with the gain chosen for the integral control as one single term shown as $\mathrm{Ki}$ in the equation for $\mathrm{n}$. Different values of $\mathrm{Ki}$ are chosen and the one with the best performance is selected.

The variable $\mathrm{n}$ is the duty cycle of the pulse-width modulated signal produced by the on-chip pulse-width modulator. In the equation for $n$, the negative of $\mathrm{Ve}$ is used because of the particular manner that the pulsewidth modulated signal operates in the Feedback mechanical unit. This particular manner was described in the previous section.

The constants 255, 0.5 and 100 in the equation for $n$ are used to scale $\mathrm{n}$ to the range of 0 to 100 percent for the case of $\mathrm{Kp}=1$ and $\mathrm{Ki}=0$. With $\mathrm{Kp}>1$ or $\mathrm{Ki}>0$, the value of $n$ can get out of the range of 0 to 100 percent. Checking was done on $\mathrm{n}$ in the $\mathrm{C}$ program. If $\mathrm{n}$ was out of range, it was brought back into the range. Since the resolution of the ATD is chosen to be eight bit. So both Vref and Vtacho are in the range of 0 to 255.

This approach was tried on the mechanical unit to control the motor running at a constant speed of 1000 rpm under the condition of constantly varying load.

Experimental results for Problem 1: the experimental result is shown in Figure 3. The motor speed signal was obtained from the tachometer. The load was kept constant first and the speed was steadily constant as observed in Figure 3. The load was then increased. That corresponded to a dip in the speed signal in Figure 3. But quickly the speed was regulated to the old speed by the embedded controller. Later the load was decreased. That corresponded to a hump in the speed signal. The speed quickly returned back to the old speed as seen in Figure 3.

It was observed that when the loading was increased, the speed suffered a droop but eventually went back to the same speed as before. When the load was decreased, the speed increased but quickly regulated back to the old speed by the controller. The embedded controller worked as expected.

Problem 2: Use the $\mathrm{HC} 12$ microcontroller as an embedded controller that drives the shaft (the output) of the mechanical unit to track the input dial. Specifically, if the input dial is rotated a certain angle, the controller should drive the output shaft to turn the same amount of angle and in the same direction as the input dial. This is a classical position servo control problem and the theoretical treatment can be easily found in the literature [6-12].

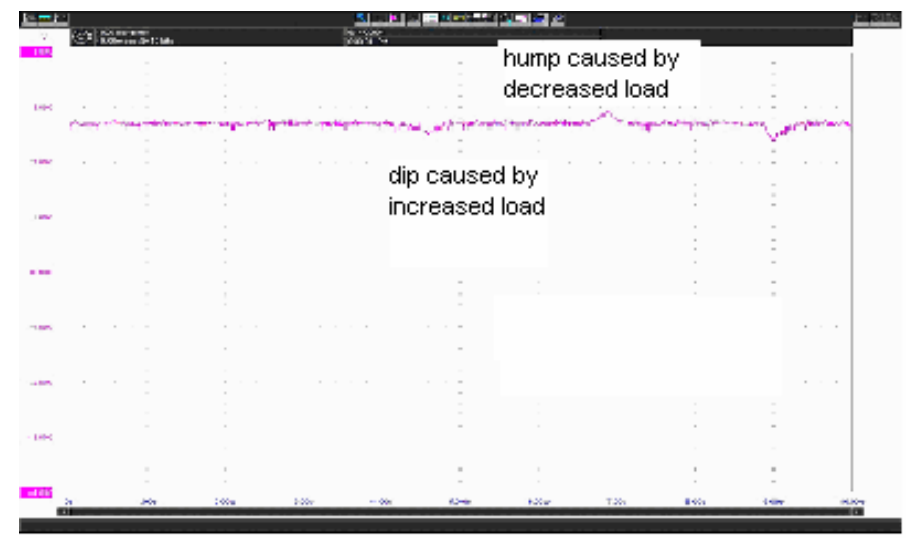

Figure 3: Experimental result for Problem 1

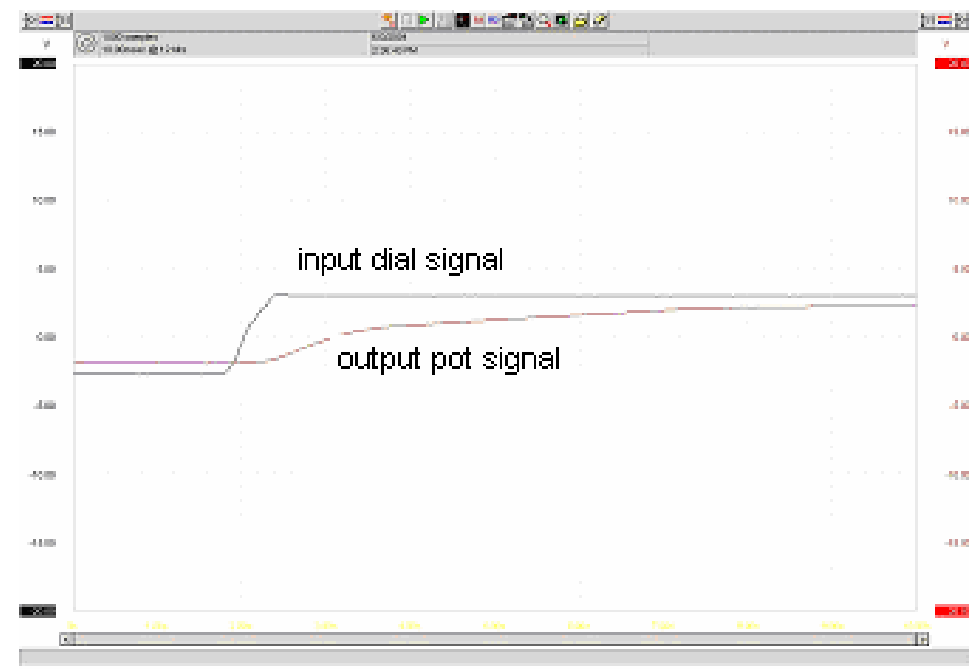

Figure 4: Experimental results for Problem 2 with gain $=1$

A solution to the problem is that the duty cycle of the pulse-width modulated signal in Figure 1 is governed by $\mathrm{K}^{*} \mathrm{Ve}$, where $\mathrm{Ve}=\mathrm{Vin}-\mathrm{Vout}$ and $\mathrm{K}$ is the proportional gain.

The proportional controller equation implemented in the $\mathrm{C}$ program are shown below. These equations are executed once every $\mathrm{T}$ seconds, where $\mathrm{T}$ is the sample time.

$\mathrm{n}=\left(-(\right.$ Vin-Vout $\left.) * \mathrm{~K}^{*} 0.5 / 255+0.5\right) * 100$

The variable $\mathrm{n}$ is the duty cycle of the pulse-width modulated signal generated by the on-chip pulse-width modulator. The variable $n$ is scaled to be within the range of 0 to 100 percent for $K=1$. The variable $n$ may go out of the range of 0 to 100 percent if $K>1$. If it was the case, $\mathrm{n}$ was brought back into range. 
Again the negative of Vin-Vout is needed because of the particular manner that the pulse-width modulated signal operates in the mechanical unit.

Experimental results for Problem 2: the experimental results for gain=1 and 5 are shown in Figures 4 and 5 . The input in each case was a step input. The input signal was generated by rotating the input dial forward a certain degrees. This input signal was the voltage from the input dial potentiometer, which was coupled to the input dial. The output shaft signal was the voltage from the potentiometer connected to the output shaft. Different values of gain were used. The effect of the magnitude of the gain was also observed. When the gain was small, the tracking was slow, with no overshoot or ringing, and with non-zero steady state error. When the gain was large enough, the tracking was much faster and came with overshoot and ringing, and with reduced steady state error.

Problem 3: Use the HC12 microcontroller as an embedded controller that drives the shaft (the output) of the mechanical unit to rotate in the direction opposite to the input dial and the amount of the angle turned by the output shaft should be the same as that of the input dial.

A solution to the problem is that the duty cycle of the pulse-width modulated signal in Figure 1 is to be proportional to the 1 's complement of the sum of $\mathrm{Vi}$ and Vo as shown in the equation below. This equation is executed once every $\mathrm{T}$ seconds, where $\mathrm{T}$ is the sample time.

$\mathrm{n}=\left(-(255-(\right.$ Vin + Vout $\left.)) * \mathrm{~K}^{*} 0.5 / 255+0.5\right) * 100$

The variable $n$ is the duty cycle of the pulse-width modulated signal generated by the on-chip pulse-width modulator. The variable $\mathrm{n}$ is scaled to be within the range of 0 to 100 percent for $K=1$. It may go out of the range of 0 to 100 percent if $K>1$. If it was the case, $n$ was brought back into range.

Experimental result for Problem 3: the experimental result is shown in Figure 6. The input signal was obtained from the input dial potentiometer. The output shaft signal was the voltage from the potentiometer connected to the output shaft. It was observed that the output shaft signal was equal in magnitude and opposite in direction to the input shaft signal. The result was as expected.

Problem 4: Use the HC12 microcontroller as an embedded controller that drives the output shaft of the mechanical unit to rotate in the direction the same as the input dial but is always at a fixed angle leading the input dial.

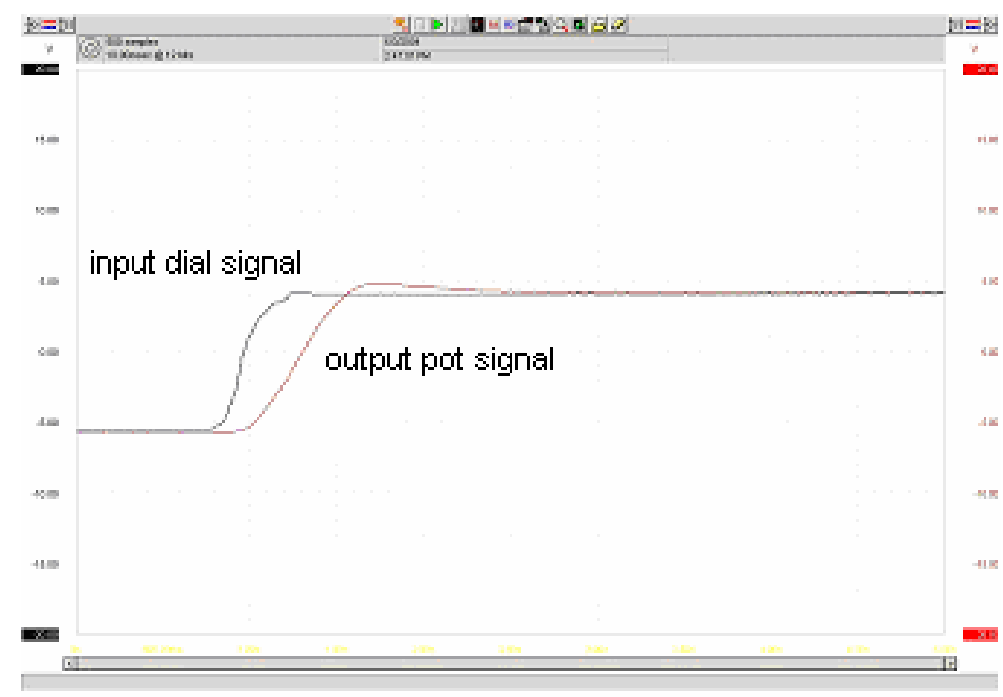

Figure 5: Experimental results for Problem 2 with gain $=5$.

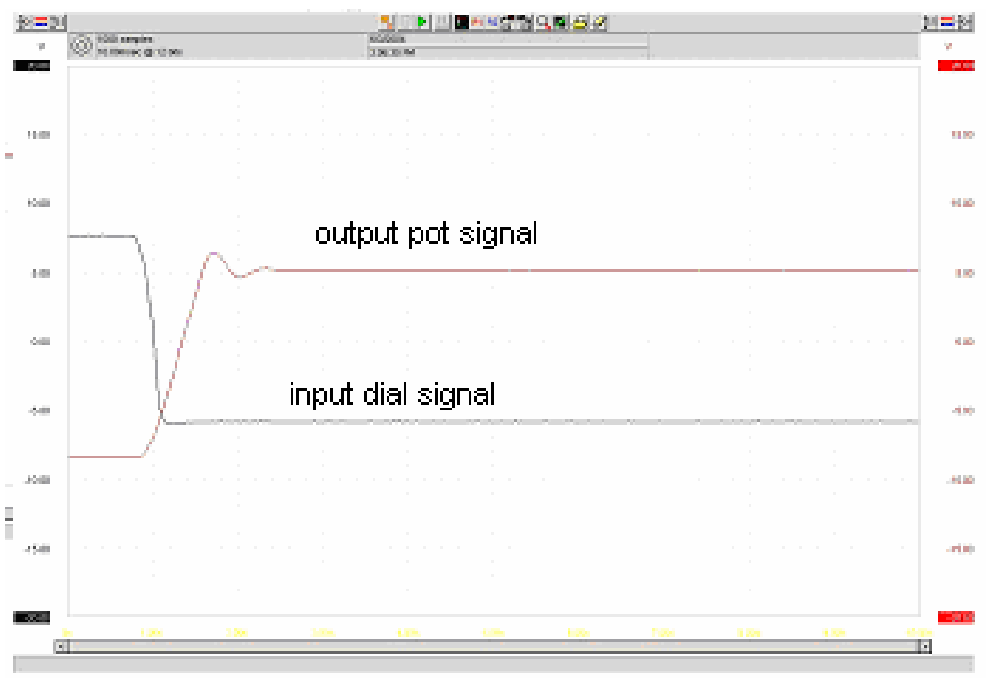

Figure 6: Experimental result for Problem 3 with gain $=20$.

A solution to the problem is that the duty cycle of the pulse-width modulated signal in Figure 1 is governed by $\mathrm{K}$ (Vin - Vout $-\mathrm{V}_{\text {fixed }}$ ), where $\mathrm{V}_{\text {fixed }}$ is a constant (binary number) that corresponds to the voltage of the fixed leading angle.

The equation for the pulse-width modulated signal duty cycle $\mathrm{n}$ in the controller $\mathrm{C}$ program is shown below. The equation is executed once every $\mathrm{T}$ seconds, where $\mathrm{T}$ is the sample time. 
$\mathrm{n}=(-($ Vin-Vout-Vfixed $) * \mathrm{~K} * 0.5 / 255+0.5) * 100$

Experimental result for Problem 4: the experimental result is shown in Figure 7 . The input signal was obtained from the input shaft potentiometer by turning the input dial. The output shaft signal was the voltage from the output shaft potentiometer. It was observed that the output shaft signal was leading the input dial signal by a constant amount. This indicates that the output shaft angle is leading the input dial angle by a fixed amount. This result was as expected.

Problem 5: Use the $\mathrm{HC} 12$ microcontroller as an embedded controller to remove the ringing in the tracking of the input shaft by the output shaft. The ringing is caused by a large proportional gain.

Velocity feedback is used to solve this problem. The velocity signal is generated by the differentiation of Vout. The duty cycle of the pulse-width modulated signal (in Figure 1) is governed by the expression

$\mathrm{K}_{1}($ Vin - Vout $)-\mathrm{K}_{2} \frac{d \text { Vout }}{d t}$

The equation for the duty cycle $\mathrm{n}$ in the controller $\mathrm{C}$ program is shown as follows:

$\mathrm{n}=-((($ Vin-Vout $) * \mathrm{~K} 1-($ Vout-Vout_old $) * \mathrm{~K} 2) / 255$

$+0.5)^{*} 100$

The equation is executed once every T seconds, where $\mathrm{T}$ is the sample time. The variable Vout_old was the value of Vout T seconds ago. The derivative is approximated by (Vout-Vout_old)/T. The parameter T is not shown explicitly in the equation for $n$. It is absorbed into K2. Different values of K2 were tried and the one with the best performance was selected.

The variable $\mathrm{n}$ is scaled to be within the range of 0 to 100 percent for $\mathrm{K} 1=1$ and $\mathrm{K} 2=0$. It may go out of the range of 0 to 100 percent if $\mathrm{K}>1$ or $\mathrm{K} 2>0$. If it was the case, $\mathrm{n}$ was brought back into range.

Experimental results for Problem 5: the experimental results are shown in Figures 8 and 9. Figure 8 shows the result with the output shaft driven by a high gain proportional controller. There was ringing. Figure 9 shows the result with the output shaft driven by the same high gain controller and with derivative feedback added. It was observed that the ringing was removed.

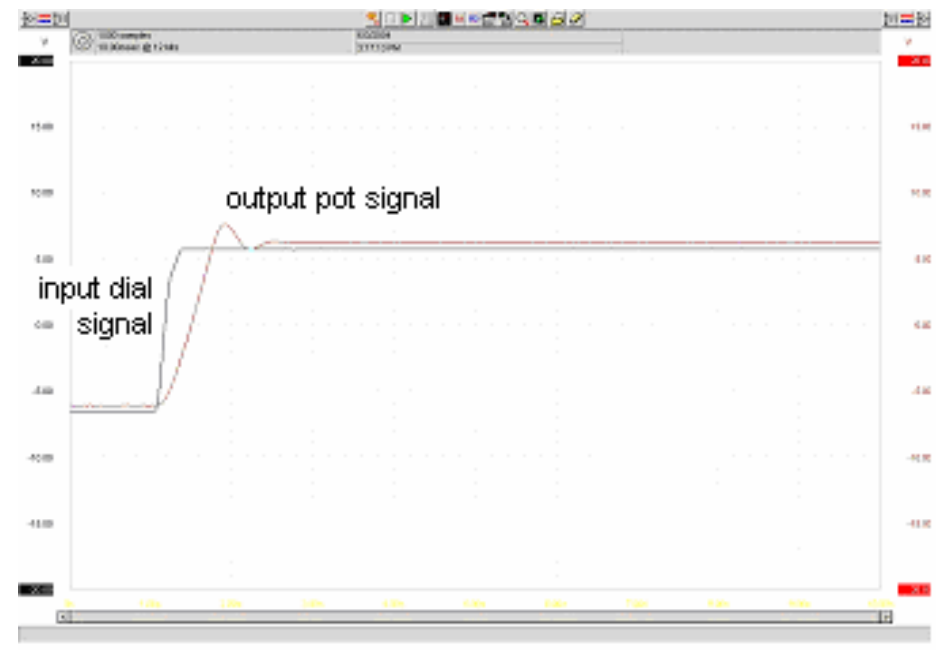

Figure 7: Experimental result for Problem 4

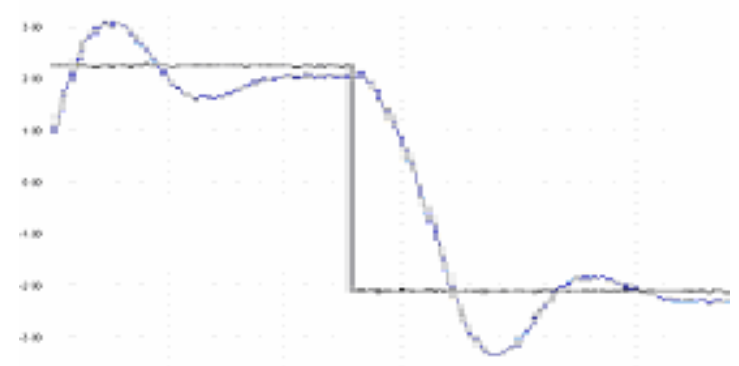

Figure 8: Experimental result for Problem 5 with high gain and no velocity feedback

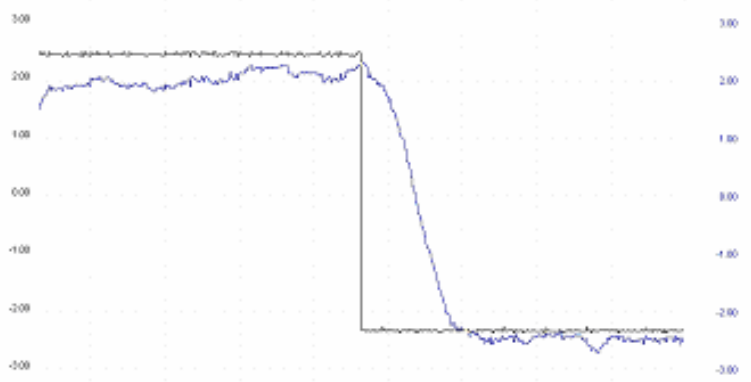

Figure 9: Experimental result for Problem 5 with high gain and with derivative feedback

\section{CONCLUDING REMARKS}

Embedded control solutions using a member of the Freescale HC12 microcontrollers to five control problems were presented and tested. The experimental results were that these embedded controllers worked as expected. These embedded solutions are among the simplest to solve the control problems without intensive learning curves. Also, simple enough are these embedded solutions that they can be easily used 
in the education of the fundamentals of embedded control principles. In programming the controller equation, attention needs to be paid to the particular manner that the plant works. In our examples, it happens that the duty cycle used the negative of the error instead of the error itself. This is due to the particular manner of the pulse-width modulation operation of the mechanical unit. Also, in the approximation of the integral and the derivative, the sample time need not be shown explicitly. It can be combined with the integral gain and the derivative gain. There are other methods [16-19] of implementation of the controllers. The comparison of the performance of these methods with the ones used in this paper will be performed in the future.

\section{REFERENCES}

[1] J.D. Aplevich, The Essentials of Linear State-Space Systems, Wiley, 2000.

[2] Astrom, et. al, DC Motor Control Trainer

Laboratory Workbook, preliminary ed., Quanser

Consulting Inc., 2004.

[3] J.S. Bay, Fundamentals of Linear State Space Systems, WCB McGraw-Hill, 1999.

[4] C.-T. Chen, Linear System Theory and Design, Oxford, 1999.

[5] C.H. Choi, "Undergraduate Controls Laboratory Experience" Proceedings of the 2004 ASEE Annual Conference, ASEE, 2004.

[6] Dorf and Bishop, Modern Control Systems, 9th Ed., Prentice Hall, 2003.

[7] Dorsey, Continuous and Discrete Control Systems, McGraw Hill, 2002.

[8] Franklin, et. al, Feedback Control of Dynamic Systems, 3rd Ed., Addison Wesley, 1994.

[9] Goodman, et al., Control System Design, Prentice Hall, 2001.

[10] Nise, Control Systems Engineering, 4th ed., John Wiley, 2004.

[11] Ogata, Modern Control Engineering, 3rd Ed., Prentice Hall, 1997.

[12] Stefani, et. al, Design of Feedback Control Systems, 4th Ed., Oxford., 2002.

[13] Nekoogar, et. al, Digital Control Using Digital Signal Processor, Prentice Hall, 1999.

[14] Thompson, Program CC v.4 Tutorial and User Guide, System Technology, Inc., 1988.

[15] Thompson, Program CC v.4 Reference Manual, System Technology, Inc., 1988.

[16] S. Tzafestas and N.P. Papanikolopoulos, "Incremental fuzzy expert PID control," IEEE Transactions on Industrial Electronics, vol. 37, no. 5, Oct. 1990 , pp. $365-371$.

[17] H.A. Malki, H. Li and G. Chen, "New design and stability analysis of fuzzy proportional-derivative control systems," IEEE Transactions on Fuzzy

Systems, vol. 2, no. 4, Nov. 1994, pp. 245 - 254 .

[18] J.E. Meng and Y. L. Sun, ’Hybrid fuzzy

proportional-integral plus conventional derivative control of linear and nonlinear systems," IEEE

Transactions on Industrial Electronics, vol. 48, no. 6, Dec. 2001 pp. 1109 - 1117.

[19] S. Seshagiri and H.K. Khalil, "Universal integral controllers with anti-reset windup for minimum phase nonlinear systems," in Proceedings of the 40th IEEE Conference on Decision and Control, 2001, vol. 5, pp. $4186-4191$.

\section{ACKNOWLEDGMENT}

Thanks to my students who assisted me in getting the experiments done. 